J. Austral. Math. Soc. 20 (Series A) (1975), 46-53.

\title{
ON A CLASS OF ANALYTIC FUNCTIONS
}

\author{
R. M. GOEL
}

(Received 15 February 1973; revised 20 May 1973)

Communicated by E. Strzelecki

\section{Introduction}

Let $S(\alpha)$ denote the class of functions

$$
f(z)=z+a_{2} z^{2}+\cdots+a_{n} z^{n}+\cdots
$$

regular and analytic in the unit disc $E=\{z:|z|<1 \mid\}$ and satisfying the condition

$$
\operatorname{Re}\left[\frac{f(z)}{z}\right]^{\frac{1}{t}}>\frac{1}{2 \alpha}, \quad \alpha \geqq 1,|z|<1 .
$$

It was shown by Robertson (1936) that if $f(z)=z+\sum_{n=2}^{\infty} a_{n} z^{n}$ is univalent and starlike in $E$ then $f(z)$ satisfies $\operatorname{Re} \sqrt{\frac{f(z)}{z}}>\frac{1}{2}$. In this paper we determine the radius of starlikeness of functions belonging to the class $S(\alpha)$.

We also obtain coefficient estimates for functions in the class $S(\alpha)$, thus generalizing a result due to Dvorak (1967).

It was further shown by Dvorak (1967) that every function $f(z)=z+\sum_{n=2}^{\infty} a_{n} z^{n}$ regular and univalent in $E$ satisfies the condition $\operatorname{Re}[f(z) / z]^{\frac{1}{2}}>\frac{1}{2}$ in a circle of radius $r_{0}$ with $0.83<r_{0}<0.84$. The exact value of $r_{0}$ has been obtained by several authors in Durren and Schober (1971), Kühnau (1971 and 1971a), Reade and Umezawa (1971). We shall find the exact value of $r_{0}(\alpha)$ for which the univalent function $f(z)=z+\sum_{n=2}^{\infty} a_{n} z^{n}$ satisfies the condition (2).

\section{Radius of starlikeness}

THEOREM 2.1. Let $f(z) \in S(\alpha)$. Let $\alpha_{0}>1$ denote the smallest positive root of the equation

$$
32 \alpha^{2}-104 x^{2}+98 \alpha-27=0 .
$$


(i) for $1 \leqq \alpha \leqq \alpha_{0}, f(z)$ is starlike in

$$
|z|<\left[\frac{8 \sqrt{4 \alpha-2}-(6 \alpha+5)}{18 \alpha-17}\right]^{\frac{1}{2}},
$$

(ii) for $\alpha \geqq \alpha_{0}, f(z)$ is starlike in

$$
|z|<\frac{\sqrt{\left(20 \alpha^{2}-28 \alpha+9\right)}-(4 \alpha-3)}{2(\alpha-1)} .
$$

These bounds are sharp.

Proof. Since $f(z) \in S(\alpha)$, we can write

$$
\sqrt{\frac{f(z)}{z}}=\frac{1}{2 \alpha}+\left(1-\frac{1}{2 \alpha}\right) p(z)
$$

where $p(z)$ is regular in $E$ and satisfies the conditions $p(0)=1$ and $\operatorname{Re} p(z)>0$ for $z \in E$. Also we know that any such function $p(z)$ can be written in the form

$$
p(z)=\frac{1-w(z)}{1+w(z)},
$$

where $w(z)$ is regular in $E$ and satisfies the conditions $w(0)=0$ and $|w(z)|<1$ for $z \in E$.

(3) and (4) yield

$$
\sqrt{\frac{f(z)}{z}}=\frac{\alpha+(1-\alpha) w(z)}{\alpha(1+w(z)}
$$

Differentiating (5) we get

$$
\frac{z f^{\prime}(z)}{f(z)}=1-\frac{2(1+A) z w^{\prime}(z)}{(1+w(z))(1-A w(z))},
$$

where $A=1-1 / \alpha, \alpha \geqq 1$. If we let $\phi(z)=w(z) / z$, then $|\phi(z)|<1$ and $\phi(z)$ is regular in $|z|<1$. Hence (Nehari (1952; page 168))

$$
\left|\phi^{\prime}(z)\right| \leqq \frac{1-|\phi(z)|^{2}}{1-|z|^{2}}
$$

Substituting for $\phi(z)$ in terms of $w(z)$ we obtain from (7)

$$
\left|z w^{\prime}(z)-w(z)\right| \leqq \frac{r^{2}-|w(z)|^{2}}{1-r^{2}}, \quad r=|z|,
$$

which, with (6) yields 
(8) $\operatorname{Re}\left[\frac{z f^{\prime}(z)}{f(z)}\right] \geqq 1-2(1+A)\left[\operatorname{Re} \frac{w(z)}{(1+w(z))(1-A w(z))}\right.$

$$
\begin{array}{r}
\left.+\frac{r^{2}-|w(z)|^{2}}{\left(1-r^{2}\right)|1+w(z)| \mid 1-A w(z)}\right] \\
=\frac{1}{1+A}\left[3 A-1+2 \operatorname{Re}\left(p(z)-\frac{A}{p(z)}\right)-\frac{2\left(r^{2}|p(z)+A|^{2}-|1-p(z)|^{2}\right)}{\left(1-r^{2}\right)|p(z)|}\right]
\end{array}
$$

where $p(z)=[1-A w(z)] /[1+w(z)], 0 \leqq A \leqq 1$.

It is easy to see that the transformation $p(z)=[1-A w(z)] /[1+w(z)]$ maps the circle $|w(z)| \leqq r$ onto the circle

$$
|p(z)-a| \leqq d, a=\frac{1+A r^{2}}{1-r^{2}}, \quad d=\frac{(1+A) r}{1-r^{2}}, \quad r=|z| .
$$

If we put $p(z)=\operatorname{Re}^{i \theta}$ and denote the right hand side of (8) by $S(R, \theta)$. Then

$$
S(R, \theta)=\frac{1}{1+A}\left[3 A-1+2 R+2\left(R-\frac{A}{R}-2 a\right) \cos \theta+\frac{2\left(a^{2}-d^{2}\right)}{R}\right]
$$

Now

$$
\frac{\partial S}{\partial \theta}=\frac{2}{1+A} \cdot \sin \theta \cdot T(R)
$$

where $T(R)=2 a+A / R-R, a-d \leqq R \leqq a+d$. Since $T(R)$ clearly is a monotone decreasing function of $R$, and since

$$
\begin{aligned}
T(a+d) & =2 \frac{1+A r^{2}}{1-r^{2}}+\frac{A(1-r)}{1+A r}-\frac{1+A r}{1-r} \\
& =\left[\frac{2\left(1+A r^{2}\right)}{1-r^{2}}-\frac{1+A r}{1-r}\right]+\frac{A(1-r)}{1+A r} \\
& =\frac{1-A r}{1+r}+\frac{A(1-r)}{1+A r}>0,
\end{aligned}
$$

It follows that $T(R)$ remains positive for $a-d \leqq R \leqq a+d$. Therefore, the maximum of $S(R, \theta)$ inside the circle $|p(z)-a| \leqq d$ is attained for $\theta=0$. By Putting $\theta=0$ in (10) we obtain

$$
S(R, 0)=\frac{1}{1+A}\left[3 A-1+2\left(2 R-\frac{A}{R}-2 a\right)+\frac{2\left(a^{2}-d^{2}\right)}{R}\right],
$$

Since

$$
a-d \leqq R \leqq a+d \text {. }
$$

$$
\begin{aligned}
& \frac{\partial S}{\partial R}=\frac{2}{1+A}\left[2+\frac{A}{R^{2}}-\frac{\left(a^{2}-d^{2}\right)}{R^{2}}\right] \\
& \quad=\frac{2}{1+A}\left[2-\frac{(1-A)\left(1+A r^{2}\right)}{1-r^{2}} \frac{1}{R^{2}}\right]=\frac{2}{1+A}\left[2-\frac{(1-A) a}{R^{2}}\right]
\end{aligned}
$$


We see that the absolute minimum of $S(R, 0)$ in $(0, \infty)$ is attained at $R=\sqrt{((1-A) a) / 2}$ and equals

$$
\frac{1}{1+A}[3 A-1+4 \sqrt{2(1-A) a}-4 a] \text {. }
$$

It is easy to see that $R_{0}<a+d$, but $R_{0}$ is not always greater than $a-d$. In such a case when $R_{0} \notin[a-d, a+d]$ the minimum of $S(R, 0)$ on the segment $[a-d$, $a+d]$ is attained at $R_{1}=a-d$ and equals

$$
\frac{1-(1+3 A) r-A r^{2}}{(1+r)(1-A r)}
$$

The two minima given by (12) and (13) coincide for such values of $A$ for which $R_{0}=R_{1}$. We thus conclude that

$$
\operatorname{Re}\left[\frac{z f^{\prime}(z)}{f(z)}\right] \geqq \frac{1}{1+A}[3 A-1-4 a+4 \sqrt{2(1-A) a}] \text { for } R_{0} \geqq R_{1},
$$

and

$$
\operatorname{Re}\left[\frac{z f^{\prime}(z)}{f(z)}\right] \geqq \frac{1-(1+3 A) r-A r^{2}}{(1+r)(1-A r)} \text { for } R_{0} \leqq R_{1} .
$$

The equality sign in (14) is attained for the function

$$
f(z)=z\left[\frac{1+(1 / \alpha-1) z}{1+z}\right]^{2} .
$$

The equality sign in (15) is attained for the function

$$
f(z)=z\left[\frac{1-1 / \alpha \cos \theta \cdot z+(1 / \alpha-1) z^{2}}{1-2 \cos \theta \cdot z+z^{2}}\right]^{2}
$$

where $\cos \theta$ is determined from

$$
\frac{1-(1+A) r \cos \theta+A r^{2}}{1-2 r \cos \theta+r^{2}}=R_{0} .
$$

Hence the radius of starlikeness for the class $S(\alpha)$ which may be obtained from (14) and (15) is given by

$$
\begin{array}{rlr}
3 A-1-4 a+4 \sqrt{2(1-A) a} & =0, & R_{0} \geqq R_{1}, \\
1-(1+3 A) r-A r^{2} & =0, A=1-1 / \alpha, R_{0} \leqq R_{1},
\end{array}
$$

which yield

$$
r_{s}=\left[\frac{8(4 \alpha-2)-(6 \alpha+5)}{18 \alpha-17}\right]^{\frac{1}{2}}, \quad R_{0} \geqq R_{1},
$$

and 


$$
r_{s}=\frac{\sqrt{\left(20 x^{2}-28 \alpha+9\right)}-(4 \alpha-3)}{2(\alpha-1)}, \quad R_{0} \leqq R_{1}
$$

The two minima given by (14) and (15) become equal to each other for such a $A(0 \leqq A<1)$ for which

$$
R_{0}=\left[\frac{(1-A) a}{2}\right]^{\frac{1}{2}}=a-d=R_{1}
$$

Hence the values of $\alpha$ for which the two values of $r_{s}$ given (20) and (21) become equal are obtained by eleminating $r$ from (19) and (22). We obtain $-27 A^{3}-17 A^{2}$ $+11 A+1=0$, and hence

$$
K(\alpha)=32 a^{3}-104 \alpha^{2}+98 \alpha-27=0,
$$

Since $K(1)=-1<0$ and $K(\infty))=+\infty$, it follows that $\alpha_{0}$ in the theorem lies in $(1, \infty)$.

The functions given by (16) and (17) show that the bounds are sharp.

\section{Coefficient estimates}

THEOREM 3.1. If $f(z)=z+a_{2} z^{2}+\cdots+a_{n} z^{n}+\cdots$ is regular and analytic in $E$ and satisfies (2), then

$$
\left|a_{n}\right| \leqq 4\left(1-\frac{1}{2 \alpha}\right)\left[n\left(1-\frac{1}{2 \alpha}\right)-\left(1-\frac{1}{\alpha}\right)\right] \text { for } n=2,3, \cdots .
$$

These bounds are sharp.

Proof. On putting $p(z)=1+\sum_{n=1}^{\infty} p_{n} z^{n}$ in (3) we get

$$
f(z)=z\left[\frac{1}{2 \alpha}+\left(1-\frac{1}{2 \alpha}\right)\left(1+\sum_{n=1}^{\infty} p_{n} z^{n}\right)\right]^{2} \text {. }
$$

On substituting the power series exapnsion for $f(z)$ from (1) in (24) and then equating the coefficients of $z^{2 m}$ and $z^{2 m+1}$ we get

$$
a_{2 m+1}=2\left(1-\frac{1}{2 \alpha}\right) p_{2 m}+\left(1-\frac{1}{2 \alpha}\right)^{2}\left(p_{m}^{2}+2 \sum p_{r} p_{s}\right), \quad r+s=2 m,
$$

and

$$
a_{2 m+2}=2\left(1-\frac{1}{2 \alpha}\right) p_{2 m+1}+2\left(1-\frac{1}{2 \alpha}\right)^{2} \sum p_{r} p_{s}, \quad r+s=2 m+1 .
$$

Since $\operatorname{Re} p(z)>0$ for $z \in E$, we have (Nehari (1952; page 170))

$$
\left|p_{n}\right| \leqq 2 \text { for } n=1,2,3, \cdots \text {. }
$$


From (25), (26) and (27) we easily obtain the bounds

$$
\begin{aligned}
\left|a_{n}\right| & \leqq 4\left(1-\frac{1}{2 \alpha}\right)\left(n-1-\frac{n-2}{2 \alpha}\right) \\
& =4\left(1-\frac{1}{2 \alpha}\right)\left[n\left(1-\frac{1}{2 \alpha}\right)-\left(1-\frac{1}{\alpha}\right)\right], \quad n=2,3, \cdots .
\end{aligned}
$$

The bounds are attained by the extremal function

$$
f(z)=z\left[\frac{1}{2 \alpha}+\left(1-\frac{1}{2 \alpha}\right) \frac{1+z}{1-z}\right]^{2} .
$$

This completes the proof of the theorem.

REMARK 1. On putting $\alpha=1$ in Theorem 3.1. we get

$$
\left|a_{n}\right| \leqq n, \quad n=2,3, \cdots,
$$

which is a result obtained by Dvorak (1967).

\section{An inequality for univalent functions}

THEOREM 4.1. Let $f(z)=z+\sum_{n=2}^{\infty} a_{n} z^{n}$ be regular and univalent in $E$. Then $f(z)$ satisfies (2) for $|z|<r_{0}(\alpha)$ where $r_{0}(\alpha)$ is the smallest positive root of the equation

(29) $\left[S^{-1}\left(\frac{1}{2} \log \frac{1+r}{1-r}\right)\right]^{2}+\left[E^{-1}\left(\frac{\sqrt{1-r^{2}}}{4 \alpha} \log \frac{1+r}{1-r}\right)\right]^{2}=\left[\frac{1}{2} \log \frac{1+\mathrm{r}}{1-r}\right]^{2}$,

where $S^{-1}(x)$ and $E^{-1}(x)$ are the inverses of $S(x)=x / \sin x$ and $E(x)=x e^{-x}$ respectively. The result is sharp.

Proof. Condition (2) is equivalent to the inequality

$$
\left|\sqrt{\frac{z}{f(z)}}-\alpha\right|<\alpha \text {. }
$$

Since $f(z)=z+\sum_{n=2}^{\infty} a_{n} z^{n}$ is regular and univalent in $E$, we have Gulusin (1947; page 113)

$$
\left|\log \sqrt{\frac{z}{f(z)}}-\frac{1}{2} \log \left(1-|z|^{2}\right)\right| \leqq \frac{1}{2} \log \left(\frac{1+|z|}{1-|z|}\right) .
$$

Putting $W=\log \sqrt{z / f}(z), \quad A=\frac{1}{2} \log \left(1-|z|^{2}\right), \quad B=\frac{1}{2} \log (1+|z|) /(1-|z|)$, $W_{1}=e^{W}=R \mathrm{e}^{i \phi}$ in (30) and (31) we obtain

$$
R<2 \alpha \cos \phi
$$

and 


$$
(\log R-A)^{2}+\phi^{2}<B^{2} \text {, }
$$

respectively.

If $|z|=r$ is small, it is evident that the region defined by (33) lies in the region (32). As $r$ increases, the boundary of (33) touches the boundary of (32) before $r$ reaches 1 . At such a point of contact we must have

$$
\log R=\log (2 \alpha \cos \phi)=\left(A+\sqrt{B^{2}-\phi^{2}}\right.
$$

and

$$
\frac{d R}{d \phi}=-2 \alpha \sin \phi=-\frac{\phi}{\sqrt{B^{2}-\phi^{2}}} \exp \left(A+\sqrt{B^{2}-\phi^{2}}\right) .
$$

On eliminating $\phi$ from (34) and (35) we get

$$
\frac{1}{2} B e^{A}=\alpha \sqrt{B^{2}-\phi^{2}} \exp \left[-\sqrt{B^{2}-\phi^{2}}\right] \text {. }
$$

From (35) and 36) we obtain

$$
\frac{\phi}{\sin \phi}=B
$$

If we denote by $E^{-1}(x)$ and $S^{-1}(x)$ the inverses of $E(x)=x e^{-x}$ and $S(x)=x / \sin x$ respectively, then (36) and (37) yield (29).

The result is sharp because the inequality (31) is sharp.

THEOREM 4.2. Let $g(z)=z+a_{3} z^{3}+\cdots$ be analytic, univalent and odd in $E$. Then $\operatorname{Re}[(g(z)) / z]>1 / 2 \alpha$ for $|z|<r_{1}(\alpha)$, where $r_{1}(\alpha)$ is the smallest positive root of the equation

$$
\left(S^{-1}\left[\frac{1}{2} \log \frac{1+\sqrt{r}}{1-\sqrt{r}}\right]\right)^{2}+\left[E^{-1}\left(\frac{\sqrt{1-r}}{4 \alpha} \log \frac{1+\sqrt{r}}{1-\sqrt{r}}\right)\right]^{2}=\left[\frac{1}{2} \log \frac{1+\sqrt{r}}{1-\sqrt{r}}\right]^{2} .
$$

The result is sharp.

Proof. If we take $f\left(z^{2}\right)=(g(z))^{2}$, then $f(z)$ is analytic and univalent in $E$ and we then apply Theorem 4.1 to obtain the above theorem.

Remark. On putting $\alpha=1$ in Theorems 4.1 and 4.2. We obtain Theorem $C$ and $D$ proved by Reade and Umezawa (1971). This shows our theorems generalize the results obtained earlier by Reade and Umezawa (1971) and Duren and Schober (1971).

The author wishes to thank the referee for his suggestions. 


\section{References}

P. L. Durren and G. E. Schober (1971), 'On a class of schlicht functions', Michigan Math. J. 18, 353-356.

Dvorák (1967), 'Ủber Schlicht Funktionen, I,' Casopis pro pěstovani matematiky 92, 162-189.

Gulusin (1957), Geometrische Funktionentheorie. (Berlin, 1957).

R. Kühnau (1971), 'Eine Bemerkung Zu Zwei Arbeiten von Herrn O. Dvorak' Casopis Pest. Math. 96, 268-269.

R. Kühnau (1971a), 'Eine Berekung zu zwei Arbeiten von O. Dorak,' Math. Nachr. 48, 225-226.

Z. Nehari (1952), Conformal Mapping. (McGraw-Hill, New York, 1952).

M. O. Reade and T. Umezawa (1971), 'An inequality for univalent functions due to Dvorak', Casopis Pěst. Mat. 96, 265-267.

M. S. Robertson (1936), 'On the theory of univalent functions,' Annals of Math. 37, 374-408.

Department of Mathematics

Punjabi University

Patiala, India 